\title{
Three risk factors for pulmonary metastasectomy in patients with hepatocellular carcinoma
}

\author{
Akifumi Nakamura ${ }^{1,2}$ (D) Minoru Esaki ${ }^{2} \cdot$ Kazuo Nakagawa $^{1} \cdot$ Keisuke Asakura ${ }^{1} \cdot$ Yoji Kishi $^{2} \cdot$ Satoshi Nara $^{2}$. \\ Kazuaki Shimada ${ }^{2}$. Shun-ichi Watanabe ${ }^{1}$
}

Received: 15 November 2018 / Accepted: 3 February 2019 / Published online: 28 February 2019

(c) The Author(s) 2019

\begin{abstract}
Objective The outcomes of surgically treating pulmonary metastases from hepatocellular carcinoma remain unclear. Therefore, we aimed to evaluate patients with hepatocellular carcinoma who underwent pulmonary metastasectomy to assess their survival outcome and prognostic factors.

Methods This retrospective single-center study included 30 patients who underwent pulmonary metastasectomy for hepatocellular carcinoma between January 1980 and December 2016 at the National Cancer Center Hospital.

Results The 1-, 3-, and 5-year overall survival rates after pulmonary metastasectomy were $86.7 \%, 46.2 \%$, and $33.6 \%$, respectively (median survival time: 25.0 months). The univariate prognostic factors were viral hepatitis $(P=0.019)$, number of pulmonary metastases $(P=0.002)$, and other site recurrence before metastasectomy $(P=0.048)$. Multivariate analysis using a Cox proportional hazards model revealed viral hepatitis (hazard ratio: $3.611,95 \%$ confidence interval: $1.226-10.64 ; P=0.02$ ) and $\geq 2$ pulmonary metastases (hazard ratio: $4.031,95 \%$ confidence interval: $1.594-10.19 ; P=0.003$ ) to be independent prognostic factors. Subgroup analyses of the three risk factors (viral hepatitis, number of pulmonary metastases, and other site recurrence before metastasectomy) revealed that the median survival times after pulmonary metastasectomy were 66.0 and 15.5 months for patients with $0-1$ risk factors and those with $2-3$ risk factors, respectively $(P<0.001)$.

Conclusions For patients who underwent pulmonary metastasectomy for hepatocellular carcinoma, median survival time was 25.0 months and decreased with three risk factors which included viral hepatitis, multiple number of pulmonary metastases, and the presence of other site recurrence before metastasectomy.
\end{abstract}

Keywords Pulmonary metastasectomy $\cdot$ Hepatocellular carcinoma $\cdot$ Survival outcome $\cdot$ Prognostic factors

\section{Introduction}

Primary hepatic cancer is an aggressive tumor with a poor prognosis and is the second leading cause of cancer-related deaths worldwide, with $>740,000$ individuals affected each year. This cancer is most prevalent in southeastern Asia and parts of Africa, and the prevalence is expected to increase in the future, with hepatocellular carcinoma (HCC) being the most common primary liver tumor in Japan. Surgery,

Akifumi Nakamura

xxmp97511@zeus.eonet.ne.jp

1 Department of Thoracic Surgery, National Cancer Center Hospital, 5-1-1, Tsukiji, Chuo-ku, Tokyo 104-0045, Japan

2 Hepatobiliary and Pancreatic Surgery Division, National Cancer Center Hospital, 5-1-1, Tsukiji, Chuo-ku, Tokyo, Japan transarterial chemoembolization, percutaneous ethanol injection, radiofrequency ablation (RFA), and chemotherapy have considerably improved the prognosis of patients with HCC. However, there is a high incidence of recurrence after hepatic resection, with Poon et al. reporting a cumulative 5-year recurrence rate of 75-100\% [1]. Although intrahepatic recurrence is the most common form, pulmonary metastases (PMs) account for $>50 \%$ of all extrahepatic metastases [2,3]. Extrahepatic metastases are also associated with a poor prognosis based on the 1-year overall survival (OS) rate of $39.3 \%$ and median survival time (MST) of 8.1 months [4].

Most patients with PMs from HCC are not suitable for surgical resection owing to their advanced disease stage, low performance status (PS), severe liver dysfunction, and presence of multiple pulmonary nodules. However, since the 2000s, several studies have assessed the efficacy 
of pulmonary metastasectomy, with Yoo et al. reporting a 5-year OS rate of $37.0 \%$ and MST of 40.7 months among 45 patients with HCC who underwent pulmonary metastasectomy [5]. Furthermore, considerable attention has been recently paid to prognostic factors among patients with $\mathrm{HCC}$ who undergo pulmonary metastasectomy. For example, Chen et al. reported that a largest PM size of $<3 \mathrm{~cm}$ was the only predictor of favorable OS $(P=0.0006)[6]$. Thus, the available reports have described variable outcomes of pulmonary metastasectomy and associated prognostic factors. The present study aimed to review our center's experience in this setting and clarify favorable indications for metastasectomy among patients with HCC.

\section{Patients and methods}

\section{Patients and data}

We retrospectively reviewed data of patients who underwent pulmonary metastasectomy for HCC at the National Cancer Center Hospital between January 1980 and December 2016. The observation period was terminated on March 31, 2017. Patients were censored on the date of death or most recent follow-up. Pulmonary metastasectomy was performed according to the following criteria: (1) no evidence of uncontrolled intrahepatic disease, (2) no evidence of extrathoracic metastasis, (3) possibility of complete pulmonary metastasectomy, (4) adequate general physical condition to permit pulmonary metastasectomy, and (5) sufficient expected pulmonary function after the pulmonary metastasectomy. These criteria were based on a modification of Thomford et al.'s principles [7]. The treatment for PM from $\mathrm{HCC}$ required multidisciplinary team involvement including hepatoma surgeons, hepatoma oncologists and thoracic surgeons.

\section{Surgical treatment and postoperative follow-up}

The surgical treatment was performed under general anesthesia with controlled ventilator support, and metastatic lesions were resected via pulmonary wedge resection, segmentectomy, or lobectomy. Wedge resection was generally performed for peripheral PM lesions, whereas anatomical resection (segmentectomy or lobectomy) was performed when the metastatic lesion was located deep in the pulmonary parenchyma. Anatomical lung resection was typically performed for intrapulmonary malignancies using a minimally invasive open surgery approach [8]. Based on the patient's size, a $6-8-\mathrm{cm}$ incision was made near the auscultatory triangle at $2 \mathrm{~cm}$ below the scapula (posterolateral thoracotomy). Dissection of the mediastinal lymph nodes was not routinely performed unless enlarged lymph nodes were noted during the preoperative examination.
The surgical outcomes were evaluated in terms of OS after the initial pulmonary metastasectomy. Patients were followed up every 1-6 months using physical examination; chest radiography; blood testing, including serum AFP levels; liver ultrasonography; lung and abdominal computed tomography (CT); and 2-deoxy-2-[ $\left[{ }^{18} \mathrm{~F}\right]$ fluoro-D-glucose position emission tomography (FDG-PET) /CT, if indicated.

\section{Clinicopathological variables and definitions}

The patients' records were retrospectively searched to obtain data regarding age at pulmonary metastasectomy, sex, presence of viral hepatitis, serum AFP level before pulmonary metastasectomy, recurrence at any site before pulmonary metastasectomy, and first recurrence site. In addition, the following surgical data were collected: number and laterality of PMs, size of the largest PM, operative method, and number of pulmonary operations. DFI was calculated as the period between the primary hepatic surgery and appearance of recurrence at any site. The OS rates were calculated from the time of the pulmonary metastasectomy to the date of the most recent follow-up or death.

\section{Statistical analysis}

Survival was estimated using the Kaplan-Meier method, and univariate prognostic analysis was performed using the log-rank test. The Cox proportional hazards model was used for the multivariate analyses, which included significant variables from the univariate analyses. Differences were considered statistically significant at $p$ values $<0.05$, and all analyses were performed using IBM SPSS software (version 22; IBM Corp., Armonk, NY, USA).

\section{Results}

\section{Patient characteristics}

Thirty patients (25 men) underwent curative pulmonary metastasectomy at our institution between January 1980 and December 2016 (Table 1). The median age at the initial pulmonary metastasectomy was 61.5 years (range 28-77 years). Sixteen patients were positive for hepatitis B virus-related antigens and 3 patients were seropositive for hepatitis $C$.

Among the 30 patients, 18 had solitary PM and 12 had multiple PMs. Twenty-three patients had unilateral PM(s) and seven had bilateral metastases. The median largest PM size was $30 \mathrm{~mm}$ (range $8-70 \mathrm{~mm}$ ). The median DFI was 18.5 months (range 0-107 months). Recurrence before pulmonary metastasectomy was noted in 22 patients, with all cases involving intrahepatic recurrence. Wedge resection was performed for 20 patients, and anatomical lung 
Table 1 Clinical characteristics of the patients

\begin{tabular}{|c|c|}
\hline Characteristics & Number \\
\hline \multicolumn{2}{|l|}{ Sex } \\
\hline Male & 25 \\
\hline Female & 5 \\
\hline \multicolumn{2}{|l|}{ Viral hepatitis } \\
\hline $\mathrm{HBV}$ & 16 \\
\hline $\mathrm{HCV}$ & 3 \\
\hline Absent & 11 \\
\hline \multicolumn{2}{|c|}{ Disease-free interval (median: 18.5 months, range $0-107$ months) } \\
\hline$<12$ & 13 \\
\hline$\geq 12$ & 17 \\
\hline \multicolumn{2}{|c|}{ Other site recurrence before metastasectomy } \\
\hline No & 22 \\
\hline Yes & 8 \\
\hline \multicolumn{2}{|c|}{ Number of pulmonary operations } \\
\hline 1 & 21 \\
\hline$\geq 2$ & 9 \\
\hline \multicolumn{2}{|c|}{ Largest PM size (median: $30 \mathrm{~mm}$, range: $8-70 \mathrm{~mm}$ ) } \\
\hline$<20 \mathrm{~mm}$ & 11 \\
\hline$\geq 20 \mathrm{~mm}$ & 19 \\
\hline \multicolumn{2}{|l|}{ Laterality of PMs } \\
\hline Right & 11 \\
\hline Left & 12 \\
\hline Bilateral & 7 \\
\hline \multicolumn{2}{|l|}{ Number of PMs } \\
\hline 1 & 18 \\
\hline$\geq 2$ & 12 \\
\hline \multicolumn{2}{|l|}{ Surgical procedure } \\
\hline Wedge resection & 20 \\
\hline Segmentectomy/lobectomy & 10 \\
\hline \multicolumn{2}{|l|}{ The year of surgery } \\
\hline Previous term (1980-1999) & 12 \\
\hline Latter term (2000-2016) & 18 \\
\hline
\end{tabular}

$H B V$ hepatitis B virus, $H C V$ hepatitis $\mathrm{C}$ virus, $P M$ pulmonary metastasis

resection (lobectomy or segmentectomy) was performed for 10 patients. No instances of in-hospital mortality were recorded.

\section{Survival}

The median follow-up after pulmonary metastasectomy was 22 months (range 1-110 months), and 21 patients died during the follow-up. At the latest follow-up, 23 patients experienced recurrence, with 19 patients having pulmonary recurrence and 9 patients undergoing repeat pulmonary metastasectomy for the newly detected nodules. MST after pulmonary metastasectomy was 25.0 months, and the 1-, 3-,

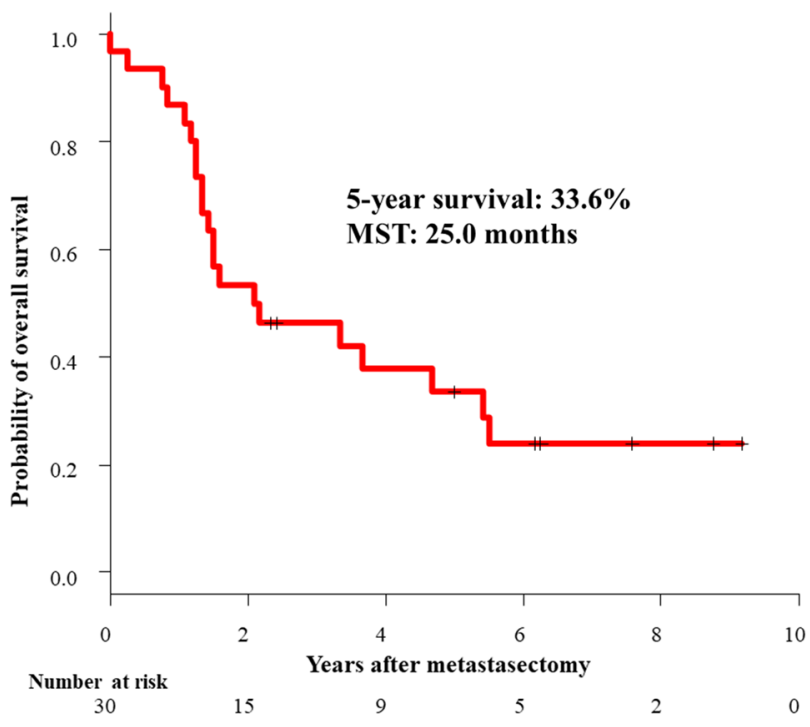

Fig. 1 Kaplan-Meier curves of overall survival after pulmonary metastasectomy for hepatocellular carcinoma. The 5-year survival rate was $33.6 \%$, and the median survival time (MST) after pulmonary metastasectomy was 25.0 months

and 5-year OS rates were $86.7 \%, 46.2 \%$, and $33.6 \%$, respectively (Fig. 1).

Univariate prognostic analysis based on Kaplan-Meier curves revealed that OS after pulmonary metastasectomy was significantly associated with viral hepatitis, number of PMs, and other site recurrence before metastasectomy. The 5-year OS was significantly higher for patients with no evidence of viral hepatitis than for those with viral hepatitis $(63.6 \%$ vs. $14.2 \% ; P=0.019)$. The 5 -year OS was significantly higher for patients with a solitary PM than for those with multiple PMs ( $46.1 \%$ vs. $16.7 \%, P=0.002)$. The OS was significantly higher for patients with no recurrence before metastasectomy than for those with other site recurrence before metastasectomy (2-year OS: $59.1 \%$ vs. $37.5 \%$, 5-year OS: $43.0 \%$ vs not reached, $P=0.048$ ) (Table 2). No other factors were associated with survival after pulmonary metastasectomy.

The multivariate analysis revealed that OS was independently predicted by viral hepatitis [hazard ratio (HR): $3.611,95 \%$ confidence interval $(\mathrm{CI}): 1.226-10.64 ; P=0.02$ ] and the number of PMs (HR: 4.031, 95\% CI: 1.594-10.19; $P=0.003$ ). Furthermore, subgroup analyses based on three risk factors, i.e., viral hepatitis, number of PMs, and other site recurrence before metastasectomy, revealed that MSTs for patients with 0 risk factors, 1 risk factor, and 2-3 risk factors were not reached at 56.0 months, and 15.5 months, respectively $(P<0.001)$ (Fig. 2; Table 3$)$. No deaths were recorded within 1 year after the pulmonary metastasectomy among patients who had 1-2 risk factors. Two patients 
Table 2 Univariate prognostic analysis using the Kaplan-Meier method

\begin{tabular}{|c|c|c|c|}
\hline Characteristics & Number & $\begin{array}{l}5 \text {-year sur- } \\
\text { vival (\%) }\end{array}$ & $p$ value \\
\hline \multicolumn{4}{|l|}{ Age (years) } \\
\hline$<65$ & 20 & 22.9 & \multirow[t]{2}{*}{0.099} \\
\hline$\geq 65$ & 10 & 58.3 & \\
\hline \multicolumn{4}{|l|}{ Sex } \\
\hline Male & 25 & 31.6 & \multirow[t]{2}{*}{0.737} \\
\hline Female & 5 & 40.0 & \\
\hline \multicolumn{4}{|l|}{ Viral hepatitis } \\
\hline Present & 19 & 14.2 & \multirow[t]{2}{*}{0.019} \\
\hline Absent & 11 & 63.6 & \\
\hline \multicolumn{4}{|l|}{ AFP } \\
\hline$<500 \mathrm{ng} / \mathrm{mL}$ & 20 & 33.9 & \multirow[t]{2}{*}{0.531} \\
\hline$\geq 500 \mathrm{ng} / \mathrm{mL}$ & 10 & 30.0 & \\
\hline \multicolumn{4}{|l|}{ Disease-free interval } \\
\hline$<12$ months & 13 & 25.6 & \multirow[b]{2}{*}{0.555} \\
\hline$\geq 12$ months & 17 & 38.8 & \\
\hline \multicolumn{4}{|c|}{ Other site recurrence before metastasectomy } \\
\hline No & 22 & 43.0 & \\
\hline Yes & 8 & NR & 0.048 \\
\hline \multicolumn{4}{|c|}{ Number of pulmonary operations } \\
\hline 1 & 21 & 41.7 & \\
\hline$\geq 2$ & 9 & 13.9 & 0.33 \\
\hline \multicolumn{4}{|l|}{ Largest PM size } \\
\hline$<20 \mathrm{~mm}$ & 11 & 47.7 & \\
\hline$\geq 20 \mathrm{~mm}$ & 19 & 25.3 & 0.067 \\
\hline \multicolumn{4}{|l|}{ Laterality of PMs } \\
\hline Unilateral & 23 & 36.1 & \\
\hline Bilateral & 7 & 28.6 & 0.202 \\
\hline \multicolumn{4}{|l|}{ Number of PMs } \\
\hline 1 & 18 & 46.1 & \\
\hline$\geq 2$ & 12 & 16.7 & 0.002 \\
\hline \multicolumn{4}{|l|}{ Surgical procedure } \\
\hline Wedge resection & 20 & 43.3 & \\
\hline Segmentectomy/lobectomy & 10 & 13.3 & 0.456 \\
\hline \multicolumn{4}{|l|}{ The year of surgery } \\
\hline Previous term (1980-1999) & 12 & 25.0 & \\
\hline Latter term (2000-2016) & 18 & 37.9 & 0.096 \\
\hline
\end{tabular}

$A F P$ alpha-fetoprotein, $N R$ not reached, $P M$ pulmonary metastasis

with all three risk factors died because of recurrence within 13 months after pulmonary metastasectomy.

\section{Discussion}

Recent improvements in surgery, chemotherapy, and interventional therapy for HCC have resulted in improved outcomes, although recurrence after surgery for HCC remains

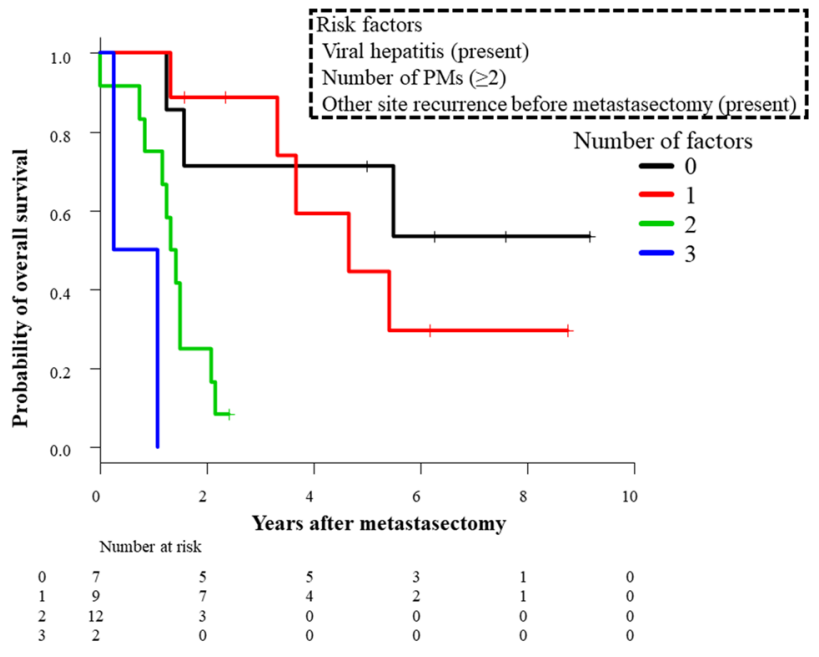

Fig. 2 Kaplan-Meier curves of overall survival after pulmonary metastasectomy for hepatocellular carcinoma according to subgroup analyses combining the three risk factors (viral hepatitis, number of PMs, and other site recurrence before metastasectomy). PM pulmonary metastasis

Table 3 Subgroup analyses combining the three risk factors: viral hepatitis, number of PMs, other site recurrence before metastasectomy

\begin{tabular}{llllll}
\hline Factors & Number & MST & $\begin{array}{l}\text { 1-year sur- } \\
\text { vival (\%) }\end{array}$ & $\begin{array}{l}\text { 3-year sur- } \\
\text { vival (\%) }\end{array}$ & $\begin{array}{l}\text { 5-year } \\
\text { survival } \\
(\%)\end{array}$ \\
\hline 0 & 7 & NR & 100 & 71.4 & 71.4 \\
1 & 9 & 56.0 & 100 & 88.9 & 44.4 \\
2 & 12 & 16.5 & 75.0 & 0 & 0 \\
3 & 2 & 8.0 & 50.0 & 0 & 0 \\
\hline
\end{tabular}

$M S T$ median survival time after pulmonary metastasectomy, $N R$ not reached, $P M$ pulmonary metastasis

common, with distant metastasis most frequently found in the lungs. There is no clear consensus regarding the optimal treatment modality for PMs in these patients, given the lack of robust evidence. Furthermore, treatment of extrahepatic metastases from HCC is complicated by the patients' low PS, severe liver dysfunction, and multiple metastases. Thus, only patients who are generally in good physical condition and have normal liver and lung functions are typically referred to the general thoracic surgery department to determine whether resection is appropriate. Nevertheless, several recent studies have assessed the efficacy of pulmonary metastasectomy and revealed 5-year OS rates that are generally in the range of $25-40 \%$ [5-10]. Our study findings are consistent with those results, suggesting that pulmonary metastasectomy is an acceptable intervention for PM from HCC. 
Other treatments for PM from HCC include RFA and molecular-targeted therapies. Li et al. [11] reported 29 unresectable cases with $58 \mathrm{PMs}$ from HCC that were treated using 51 RFA sessions and revealed that 1-, 3-, and 5-year OS rates after the initial RFA session were $71.6 \%, 27.9 \%$, and $9.3 \%$, respectively. In that study, MST was 26.3 months (range 3-66 months). Sorafenib therapy is the current standard of care for advanced unresectable HCCs because this multikinase inhibitor has proven efficacy against HCC that highlights the potential of molecular-targeted therapies in this setting. The Sorafenib Hepatocellular Carcinoma Assessment Randomized Protocol trial was the first prospective, randomized, placebo-controlled trial to confirm a survival benefit among patients with HCC based on MST values of 10.7 months in the sorafenib group and 7.9 months in the placebo group (HR: $0.68,95 \%$ CI: $0.55-0.87 ; P<0.001$ ) [12].

Among patients with good general condition, normal liver and lung functions, and potentially resectable disease, the pulmonary metastasectomy was reported to improve survival $[6,10]$. In contrast, RFA and sorafenib therapy are typically used for patients with low PS and/or severe liver and lung dysfunction because surgery is inadvisable for these patients. Nevertheless, the favorable results in the surgery group undoubtedly reflect a selection bias in terms of tumor burden and biology. Thus, to minimize the influence of a selection bias, Tomimaru et al. compared resection and non-resection groups of patients with similar clinicopathological factors, which revealed 3-year OS rates of $33.3 \%$ in the resection group and $0 \%$ in the non-resection group [13]. Furthermore, pulmonary metastasectomy has an acceptable safety profile because the 2014 annual report from The Japanese Association for Thoracic Surgery indicated that the 30-day postoperative mortality rate was only $0.2 \%$ [14]. Moreover, the present study did not detect any cases with major complications and there was no 30- and 90-day mortality. Thus, pulmonary metastasectomy may be acceptable for patients with good PS.

To the best of our knowledge, there are limited data regarding factors that predict prognosis after pulmonary metastasectomy for HCC. Takahashi et al. reported that a DFI of $\geq 12$ months was significantly associated with favorable rates of 5 -year OS $(59.3 \%$ vs. $28.7 \% ; P=0.026)$ and 5 -year disease-specific survival $(62.5 \%$ vs. $36.2 \% ; P=0.038)$ after pulmonary metastasectomy [10]. Kawamura et al. also reported that having $\leq 3 \mathrm{PMs}$ was the only factor to independently predict a good prognosis $(P=0.048)$ [15], whereas Nakagawa et al. reported that a disease-free interval (DFI) of $>1$ year and serum alfa-fetoprotein (AFP) levels of $<500 \mathrm{ng} /$ $\mathrm{mL}$ were associated with good outcomes after pulmonary metastasectomy for HCC $(P=0.015)$ [16]. In addition, Ohba et al. reported that patients with AFP levels $\geq 500 \mathrm{ng} / \mathrm{mL}$ had significantly worse rates of OS and cancer-specific survival than patients with AFP levels $<500 \mathrm{ng} / \mathrm{mL}(P<0.05)$ [17]. Thus, although several small studies have evaluated pulmonary metastasectomy for HCC, they revealed different recommended indications and prognostic factors. Although the present study evaluated a relatively small number of patients, our results confirmed that OS was significantly related to viral hepatitis, number of PMs, and other site recurrence before pulmonary metastasectomy. Based on our results, the 5 -year OS rates were $63.6 \%$ for patients without viral hepatitis and $14.2 \%$ for patients with viral hepatitis $(P=0.019)$, and we are unaware of any other reports that have described this relationship. Nevertheless, Nishikawa et al. implicated various non-hepatitis-related factors (e.g., nonalcoholic fatty liver disease as the hepatic manifestation of metabolic syndrome) in the development of HCC (i.e., non-B non-C $\mathrm{HCC}$ ) and reported that patients with non-B non-C HCC who underwent curative therapy exhibited clinical outcomes comparable or superior to those of patients with hepatitisrelated HCC [18].

Several studies have demonstrated that DFI is a prognostic factor. However, the present study revealed no significant difference in 5-year OS between 13 patients with DFI $<12$ months and 17 patients with DFI $\geq 12$ months (25.6\% vs. $38.8 \% ; P=0.555)$.

The present study also included subgroup analyses based on the patients' number of poor prognostic factors (presence of hepatitis, number of PMs, and other site recurrence before metastasectomy), and we believe that no other clinical studies have used this scoring system to assess prognostic factors among patients who underwent pulmonary metastasectomy for HCC. The 5-year OS rate among the 16 patients with $0-1$ risk factors was $59.1 \%$, and their MST was 66.0 months, which was significantly better than the rate among the 14 patients with 2-3 risk factors (MST: 15.5 months, $P<0.001)$. Interestingly, 13 of those 14 patients experienced recurrence after pulmonary metastasectomy. These findings suggest that pulmonary metastasectomy should be avoided for patients with $2-3$ of those risk factors, although it might be appropriate for patients with $0-1$ risk factors based on their relatively good outcomes. In addition, pulmonary metastasectomy was safe and had a low rate of complications, which might facilitate more effective subsequent treatments.

The present study had several limitations. First, the small sample size $(n=30)$ and retrospective single-center design are associated with risks of bias, and the results should be carefully interpreted. Nevertheless, other recent studies regarding prognostic factors associated with pulmonary metastasectomy for HCC have also been limited by their small study populations. A second limitation is that most patients were selected for surgery based on their PS plus lung and liver functions. Further studies are required to elucidate other prognostic factors that may aid in the surgical 
indication and prediction of oncological outcomes. A third limitation is that the time period spans several decades. As such, understanding control of disease has improved significantly with the routine use of CT and FDG-PET-CT. In addition, the treatment modalities for viral hepatitis and the use of chemotherapy target agents have changed significantly. To solve this problem, we performed analysis of the year of surgery. There were no significant differences between the previous term and the latter term (Table 2). However, the results should be carefully interpreted, with further work remains to be done in this field.

\section{Conclusion}

For patients who underwent pulmonary metastasectomy for HCC, MST was 25.0 months and decreased with three risk factors; viral hepatitis, number of PMs, and other site recurrence before metastasectomy. Nevertheless, further large-scale studies are needed to determine the effects of pulmonary metastasectomy on patients' prognosis and related prognostic factors.

\section{Compliance with ethical standards}

Conflict of interest The authors have no conflicts of interest.

Open Access This article is distributed under the terms of the Creative Commons Attribution 4.0 International License (http://creativeco mmons.org/licenses/by/4.0/), which permits unrestricted use, distribution, and reproduction in any medium, provided you give appropriate credit to the original author(s) and the source, provide a link to the Creative Commons license, and indicate if changes were made.

\section{References}

1. Poon RT, Fan ST, O’Suilleabhain CB, Wong J. Aggressive management of patients with extrahepatic and intrahepatic recurrences of hepatocellular carcinoma by combined resection and locoregional therapy. J Am Coll Surg. 2002;195:311-8.

2. Ikai I, Arii S, Kojiro M, Ichida T, Makuuchi M, Matsuyama Y, et al. Reevaluation of prognostic factors for survival after liver resection in patients with hepatocellular carcinoma in a Japanese nationwide survey. Cancer. 2004;101:796-802.

3. Natsuizaka M, Omura T, Akaike T, Kuwata Y, Yamazaki K, Sato $\mathrm{T}$, et al. Clinical features of hepatocellular carcinoma with extrahepatic metastases. J Gastroenterol Hepatol. 2005;20:1781-7.

4. Uchino K, Tateishi R, Shiina S, Kanda M, Masuzaki R, Kondo Y, et al. Hepatocellular carcinoma with extrahepatic metastasis: clinical features and prognostic factors. Cancer. 2011;117:4475-83.

5. Yoo YS, Kim HK, Kim J, Choi YS, Shim YM, Paik SW, et al. Long-term survival and prognostic factors after pulmonary metastasectomy in hepatocellular carcinoma. Ann Surg Oncol. 2010;17:2795-801.

6. Chen F, Sato K, Fujinaga T, Sonobe M, Shoji T, Sakai H, et al. Pulmonary resection for metastases from hepatocellular carcinoma. World J Surg. 2008;32:2213-7.

7. Thomford NR, Woolner LB, Clagett OT. The surgical treatment of metastatic tumors in the lungs. J Thorac Cardiovasc Surg. 1965;49:357-63.

8. Asamura $\mathrm{H}$. Minimally invasive open surgery approach for the surgical resection of thoracic malignancies. Thorac Surg Clin. 2008;18:269-73.

9. Koide N, Kondo H, Suzuki K, Asamura H, Shimada K, Tsuchiya R. Surgical treatment of pulmonary metastasis from hepatocellular carcinoma. Hepatogastroenterology. 2007;54:152-6.

10. Takahashi Y, Ikeda N, Nakajima J, Sawabata N, Chida M, Horio $\mathrm{H}$, et al. Prognostic analysis of surgical resection for pulmonary metastasis from hepatocellular carcinoma. World J Surg. 2016;40:2178-85.

11. Li X, Wang J, Li W, Huang Z, Fan W, Chen Y, et al. Percutaneous CT-guided radiofrequency ablation for unresectable hepatocellular carcinoma pulmonary metastases. Int J Hyperthermia. 2012;28:721-8.

12. Llovet JM, Ricci S, Mazzaferro V, Hilgard P, Gane E, Blanc JF, et al. Sorafenib in advanced hepatocellular carcinoma. N Engl J Med. 2008;359:378-90.

13. Tomimaru Y, Sasaki Y, Yamada T, Eguchi H, Takami K, Ohigashi $\mathrm{H}$, et al. The significance of surgical resection for pulmonary metastasis from hepatocellular carcinoma. Am J Surg. 2006; $192: 46-51$

14. Committee for Scientific Affairs, Japanese Association for Thoracic Surgery. Masuda M, Okumura M, Doki Y, Endo S, et al. Thoracic and cardiovascular surgery in Japan during 2014. Annual report by The Japanese Association for Thoracic Surgery. Gen Thorac Cardiovasc Surg 2016;64:665-697

15. Kawamura M, Nakajima J, Mitsuguma H, Horio H, Miyoshi S, Nakagawa K, et al. Surgical outcomes for pulmonary metastases from hepatocellular carcinoma. Eur J Cardiothorac Surg. 2008;34:196-9.

16. Nakagawa T, Kamiyama T, Nakanishi K, Yokoo H, Kamachi H, Matsushita M, et al. Pulmonary resection for metastases from hepatocellular carcinoma: factors influencing prognosis. J Thorac Cardiovasc Surg. 2006;131:1248-54

17. Ohba T, Yano T, Yoshida T, Kawano D, Tsukamoto S, Shoji F, et al. Results of a surgical resection of pulmonary metastasis from hepatocellular carcinoma: prognostic impact of the preoperative serum alpha-fetoprotein level. Surg Today. 2012;42:526-31.

18. Nishikawa H, Osaki Y. Non-B, non-C hepatocellular carcinoma (Review). Int J Oncol. 2013;43:1333-42.

Publisher's Note Springer Nature remains neutral with regard to jurisdictional claims in published maps and institutional affiliations. 